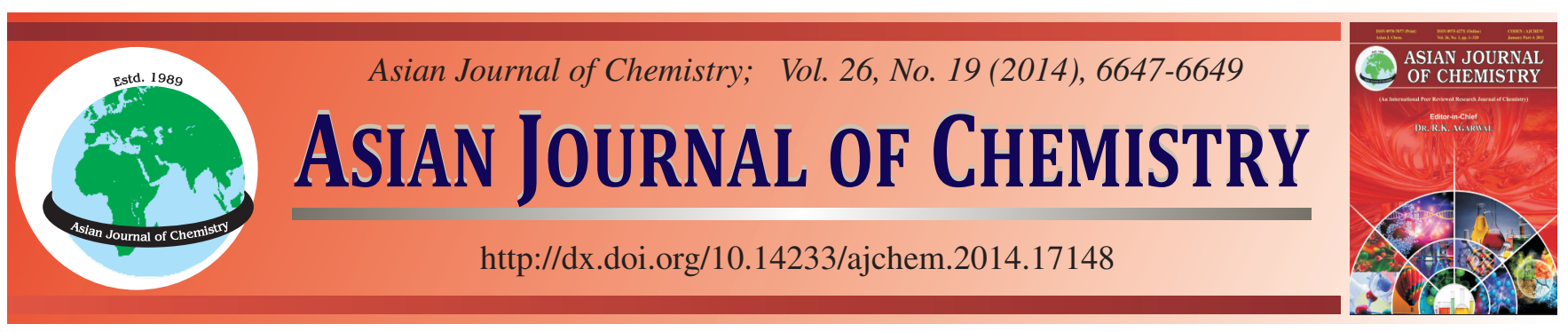

\title{
Photosynthetic Bacteria VOTO1-G Isolated from River Sediment and its Removal Capacity of Nitrogen and Phosphorus
}

\author{
Lin Wang ${ }^{1}$, Pengyan Zhang ${ }^{1}$, Meng ZhaO ${ }^{1}$ and $\mathrm{JI}_{\mathrm{LI}}{ }^{2, *}$
}

${ }^{1}$ College of Environment and Planning, Henan University, Kaifeng, Henan, P.R. China

${ }^{2}$ Department of Ecology and Environmental Sciences, College of Resource and Environment, China Agricultural University, Beijing, P.R. China

*Corresponding author: Tel: +86 10 62732017; E-mail: liji@ cau.edu.cn

\begin{abstract}
One strain of photosynthetic bacteria, named VOTO1-G, was isolated from river sediment by using of enrichment culture medium and further was used to test its role in the removing of nitrogen and phosphorus from wastewater in laboratory. By checking the individual morphology, colony culture characteristics, DNA sequencing and 16S rDNA gene bank, VOTO1-G was identified as Rhodopseudomonas palustris. The removal of total nitrogen $(50 \%)$ and total phosphorus $(78 \%)$ were observed in a 25 days period with an addition of VOTO1-G at a rate of $0.1 \%$ (v/v). It is indicated that the screened VOTO1-G strain has the capability of purifying wastewater.
\end{abstract}

Keywords: Photosynthetic bacteria, Eutrophication, River sediment, Domestic wastewater, Bioremediation.

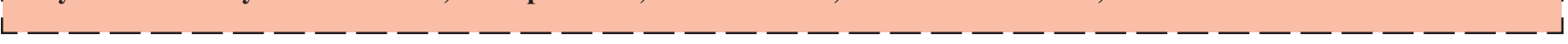

\section{INTRODUCTION}

In recent years, many researches are reported that photosynthetic bacteria can remove nutrients from wastewater ${ }^{1-4}$. The scientific literature is focused on the development of biological removal new technology, new concept, strain isolation and other aspects. Phototrophic bacteria (purple non-sulfur bacteria) are ubiquitous in fresh and marine water, soil, wastewater and activated sludge. They are metabolically the most nutrients, so they have been used for treatment of many types of wastes $e . g$., agricultural waste ${ }^{5}$, concentrated latex wastewater $^{3}$, aquarium wastewater ${ }^{6}$ and odorous swine wastewater treatment ${ }^{7}$. So far, a variety of different types of photosynthetic bacteria have been found, but different photosynthetic bacteria have the different nitrogen and phosphorus removal capacity. So screening of efficient degrading strains is still a hot research.

The main focus of the study is to isolate microorganisms of efficient degradation strains of photosynthetic bacteria. To gain efficient degradation strains of photosynthetic bacteria, we sampled sediment from the river received sewage. Such microorganism is expected to be of a source organism for purifying wastewater and is important to treat domestic wastewater.

\section{EXPERIMENTAL}

Isolations and cultivations: Bejing Qinghe received sewage, is characterized by nitrogen, phosphorus content is very high, the total nitrogen concentration of $9.46 \mathrm{mg} / \mathrm{L}$ (as
N) and total phosphorus concentration of $0.96 \mathrm{mg} / \mathrm{L}(\mathrm{P})$. Sediment was collected Bejing Qinghe sewage using a sterile bailer and filled in a sterile plastic bottle $(50 \mathrm{~mL})$, immediately frozen and transported to the laboratory for analysis.

For enrichment and culture, a liquid medium was prepared for photosynthetic bacteria (per liter): $1 \mathrm{~g}$ sodium acetate, $1 \mathrm{~g}$ sodium propionate, $1 \mathrm{~g}$ sodium butyrate, $2.7 \mathrm{~g}$ DL-malic acid, $0.5 \mathrm{~g} \mathrm{~K}_{2} \mathrm{HPO}_{4}, 0.5 \mathrm{~g} \mathrm{KH}_{2} \mathrm{PO}_{4}, 0.1 \mathrm{gMgSO}_{4} \cdot 7 \mathrm{H}_{2} \mathrm{O}, 0.8 \mathrm{~g}$ $\left(\mathrm{NH}_{4}\right)_{2} \mathrm{HPO}_{4}, 27 \mathrm{mg} \mathrm{CaCl} \cdot 2 \mathrm{H}_{2} \mathrm{O}, 1.2 \mathrm{mg} \mathrm{MnSO} \cdot 5 \mathrm{H}_{2} \mathrm{O}, 1 \mathrm{mg}$ nicotinic acid, $1 \mathrm{mg}$ vitamin $\mathrm{B}_{1}$ and $0.01 \mathrm{mg}$ biotin, $\mathrm{pH} 6.8$ and solidified with $2 \%$ agar for plate culture ${ }^{7}$. The medium was sterilized by autoclaving for $20 \mathrm{~min}$ at $121{ }^{\circ} \mathrm{C}$ and 1.1 MPa before inoculation and anaerobically cultured. Firstly, $50 \mathrm{~mL}$ sediment sample was inoculated into 1-L sterile medium at $30{ }^{\circ} \mathrm{C}$ for $7 \mathrm{~d}$ to enrich without shaking and illuminated with a light intensity of 4000 lux. Isolates were then cultivated at $30{ }^{\circ} \mathrm{C}$ on solid medium by plate serial dilutions for many times until purification.

Identification: Cells were collected after incubation in screw-capped test tubes $(16 \times 100 \mathrm{~mm})$ and chromosomal DNA was extracted and purified using benzyl chloride method $^{8}$. In vitro amplification of extracted 16s rDNA was done as previously described by Yoon et al. ${ }^{9}$ with universal primers 9F and $1512 \mathrm{R}$. The $16 \mathrm{~S}$ rDNA partial sequences were joined using SeqMan software and blasted in Genbank.

Nitrogen and phosphate removal: Eutrophicated water was collected from Bejing Qinghe, China, in plastic buckets. The water was then placed in a laboratory or a greenhouse. In 
August, the experiments were carried out in a greenhouse at China Agricultural University. Six plastic buckets filled with $20 \mathrm{~L}$ of wastewater were prepared. Three buckets were treated with VOTO1-G suspension liquid and three buckets were treated with aeration only (controls). Wastewater was inoculated with $0.1 \%$ (v/v) of VOTO1-G suspension liquid in each $20 \mathrm{~L}$ wastewater sample once every 5 days. They were incubated in a rotary shaker $(150 \mathrm{rpm})$ for 5 days after inoculation. Water samples were collected in plastic bottles for water quality analyses both before and after inoculation. All samples were stored at $-4{ }^{\circ} \mathrm{C}$ if not analyzed immediately.

Water quality analyses were conducted using standard methods ${ }^{10}$. The colourimetric method was used for total nitrogen (TN), total phosphorus (TP). For each parameter, duplicate samples were analyzed. To evaluate the effect of inoculation with VOTO1-G we used a one-way ANOVA (SPSS, release 11.5) for each parameter.

\section{RESULTS AND DISCUSSION}

Isolation and Identification of purple non-sulfur bacteria: The study aimed at the strains with the capability of removing nitrogen and phosphate. Under the conditions used in the experiments, purple non-sulfur bacteria, strain VOTO1$\mathrm{G}$ was purified by traditional plate isolation and its colony was shown in Fig. 1. The strain was examined microscopically (Fig. 2). Then the strain VOTO1-G was isolated and selected as the strain for further studies on its capability of removal of nitrogen and phosphorus.

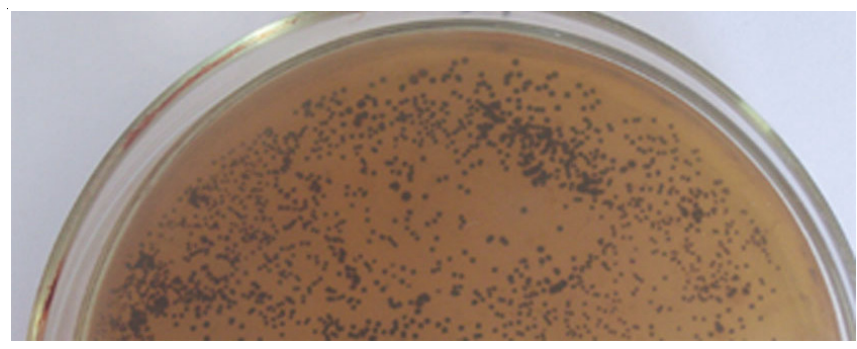

Fig. 1. Colony of strain VOTO1-G

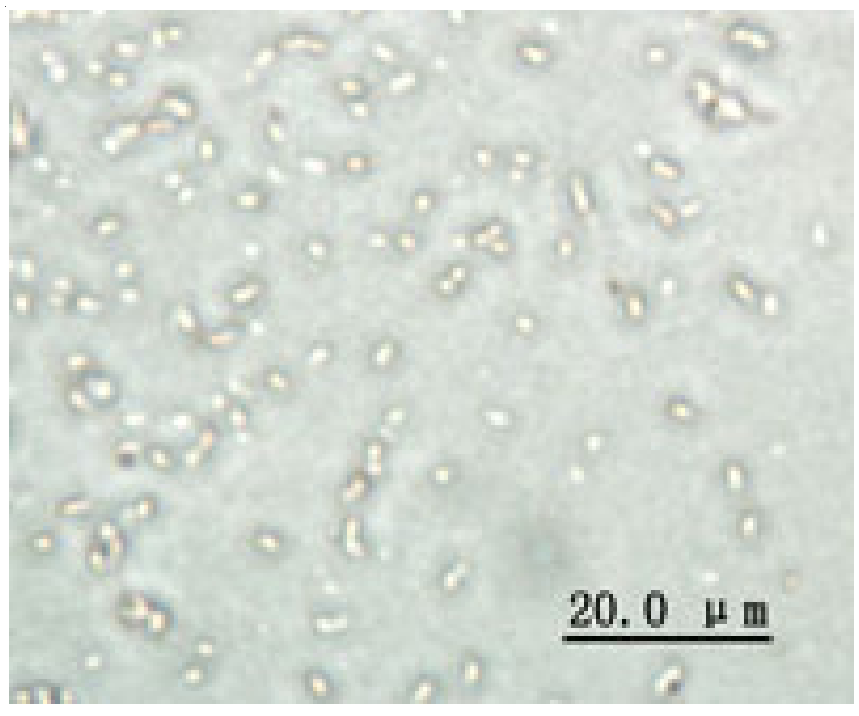

Fig. 2. Light micrograph of strain VOTO1-G showing cell shape
Strain VOTO1-G was a Gram-negative, catalase-positive, non-spore-forming, motile rod $(0.6-0.9 \times 1.2-2.0 \mu \mathrm{m})$, was red. Its identity was confirmed by standard method and its 16S rDNA sequence (1375bp), when compared with all accessible 16S rDNA sequence in the GenBank database, showed a $100 \%$ match with the sequence of Rhodopseudomonas palustris ATCC (D25312). The NCBI GenBank accession number for the $16 \mathrm{~S}$ rDNA sequence of the strain VOTO1-G is FJ695508.

Total nitrogen removal: For total nitrogen, the reduction in total nitrogen was higher in the inoculated treatment than in the control (Fig. 3). It showed that strain VOTO1-G had the capacity of nitrogen removal. The total nitrogen concentration in the treatments changed greatly with time. Removal rates were significantly higher in the inoculated treatment. The lack of significant improvement in removal rate over time may be due to fixing of atmospheric nitrogen either by free-living or symbiotic organisms ${ }^{11-13}$. During the first 5 days, this result of total nitrogen in the inoculated treatment was nearly identical to that in the control, because of added strain VOTO1-G in the acclimatization stage and the removal of nitrogen being the role of indigenous microorganisms in water bodies. With the addition of VOTO1-G, the nitrogen removal rate was obvious. After 25 days, total nitrogen removal in the inoculated treatment reached $50 \%$ and was significantly higher than in the control.

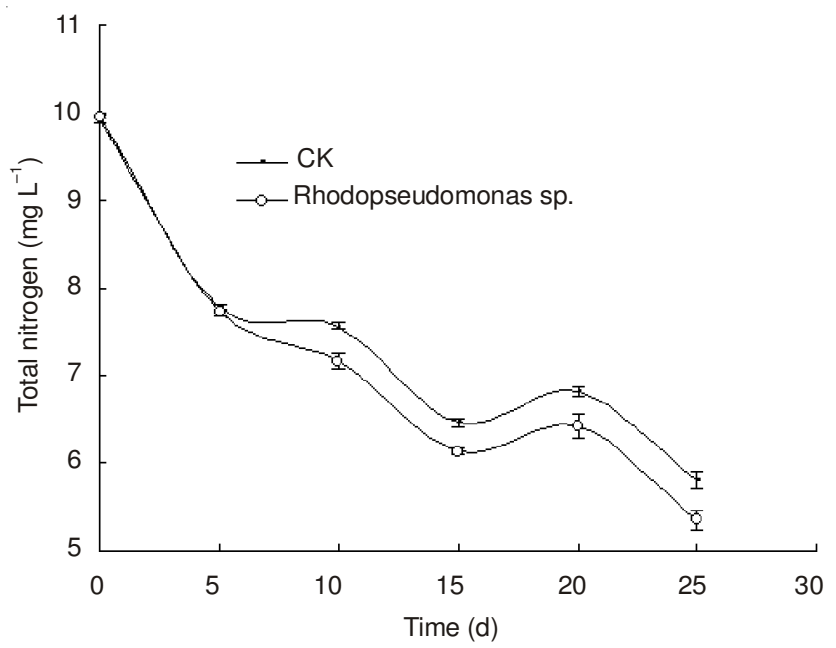

Fig. 3. Change in total nitrogen concentration over time in the two treatments. Error bars indicate one standard deviation, based on three replicates

Total phosphate removal: For total phosphate, the total phosphorus removal with and without strain VOTO1-G are shown in Fig. 4. During the test, total phosphorus concentration in the inoculated treatment was significantly lower than in the control. The result revealed that strain VOTO1-G had the capacity of phosphorus removal. In the first 5 days, compared with the control phosphorus removal effect is not obvious. Lack of significant improvement in removal rate may be due to added strain VOTO1-G in the acclimatization stage and the removal of phosphorus being the role of indigenous microorganisms in water bodies. total phosphorus levels in the treatments decreased sharply in the first 10 days of treatment but did not decrease much in the subsequent 15 days. 
The result revealed that total phosphorus removal was significantly higher in the inoculated buckets $(78 \%)$ than in the control buckets after 25 days. This result of total phosphorus in wastewater was nearly identical to that in odorous swine wastewater treatment using Rhodopseudomonas palustris reported by Kim et al. ${ }^{7}$.

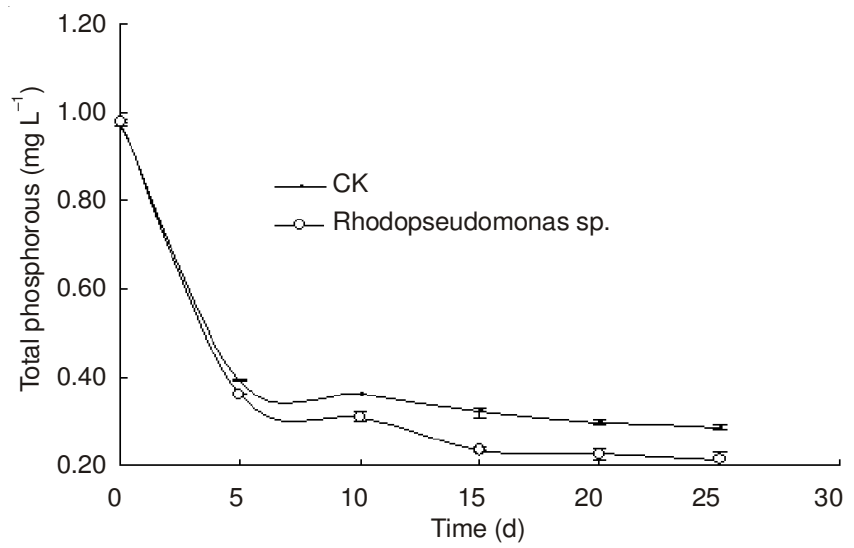

Fig. 4. Change in total phosphorus concentration over time in the two treatments. Error bars indicate one standard deviation, based on three replicates

\section{Conclusions}

- Using an enrichment culture and plate dilution method, one strain of photosynthetic bacteria associated with highly removal capability of pollutant, named VOTO1-G was successfully isolated from sewage sediments. By checking the individual morphology, colony culture characteristics, $16 \mathrm{~S}$ rDNA sequencing, VOTO1-G was identified as Rhodopseudomonas palustris.

- The VOTO1-G bacteria was researched its ability in removing of nitrogen and phosphorus from eutrophic water by $0.1 \%$, which removed total nitrogen $50 \%$ and total phosphorus $78 \%$. It indicated that its removal capacity of nitrogen and phosphorus was good and provided microbiology foundation for development of urban sewage nutrient removal technology.
- The nutrient removal characteristics of bacteria are of particular value for in situ bioremediation. In order to achieve the economic and effective method, further study should be carried out to explore the linear relationship between the addition quantity of VOTO1-G and the extent of eutrophication.

\section{ACKNOWLEDGEMENTS}

This work was supported by Ecological Research Program of Beijing, China (Fund number: XK10019440), the Education Department of Henan Province Natural Science Research Project (Fund number: 2011B610003) and Post-doctoral Foundation of China (Fund number: 2012M511110). Special thanks are due to the reviewers for their valuable comments on the manuscript

\section{REFERENCES}

1. G.Y. Jung, H.O. Jung, J.R. Kim, Y. Ahn and S. Park, Biotechnol. Lett., 21, 525 (1999)

2. H. Nagadomi, T. Kitamura, M. Watanabe and K. Sasaki, Biotechnol. Lett., 22, 1369 (2000).

3. W. Choorit, P. Thanakoset, J. Thongpradistha, K. Sasaki and N. Noparatnaraporn, Biotechnol. Lett., 24, 1055 (2002).

4. C.M. Liang, C.H. Hung, S.C. Hu and I.C. Ye, Appl. Microbiol. Cell Physiol., 86, 709 (2010).

5. A. Hiraishi, J.L. Shi and H. Kitamura, J. Ferment. Bioeng., 68, 269 (1989).

6. H. Nagadomi, T. Hiromitsu, K. Takeno, M. Watanabe and K. Sasaki, J. Biosci. Bioeng., 87, 189 (1999).

7. M.K. Kim, K.-M. Choi, C.-R. Yin, K.-Y. Lee, W.-T. Im, J.H. Lim and S.-T. Lee, Biotechnol. Lett., 26, 819 (2004).

8. H. Zhu, F. Qu and L.H. Zhu, Nucleic Acids Res., 21, 5278 (1993).

9. J.H. Yoon, S.T. Lee and Y.H. Park, Int. J. Syst. Bacteriol., 48, 187 (1998).

10. APHA, American Public Health Association, Washington, DC, edn, 18 (1992).

11. A. Watanabe and Y. Yamamoto, Nature, 214, 738 (1967).

12. B. Bergman, J.R. Gallon and A.N. Rai, FEMS Microbiol. Rev., 19, 139 (1997).

13. D.P. Hader, H.D. Kumar, R.C. Smith and R.C. Worrest, J. Photochem. Photobiol. B, 46, 53 (1998). 